\title{
Féeries
}

Études sur le conte merveilleux, XVII $-\mathrm{XIX}{ }^{\mathrm{e}}$ siècle

Contes et morale(s)

\section{L'interdit salutaire à travers deux cas merveilleux : "Cendrillon » (Perrault) et « Les six cygnes » (Grimm)}

Salutary Forbidden Precepts in Perrault's "Cinderella" and the Grimms' "Six Swans"

Pierre-Emmanuel Moog

\section{OpenEdition}

Journals

Édition électronique

URL : http://journals.openedition.org/feeries/998

ISSN : 1957-7753

Éditeur

UGA Éditions/Université Grenoble Alpes

Édition imprimée

Date de publication : 15 octobre 2016

Pagination : 87-115

ISBN : 978-2-8310-335-3

ISSN : 1766-2842

Référence électronique

Pierre-Emmanuel Moog, «L'interdit salutaire à travers deux cas merveilleux : « Cendrillon » (Perrault) et "Les six cygnes » (Grimm) », Féeries [En ligne], 13| 2016, mis en ligne le 01 janvier 2017, consulté le 08 septembre 2020. URL : http://journals.openedition.org/feeries/998 


\section{L'INTERDIT SALUTAIRE À TRAVERS DEUX CAS \\ MERVEILLEUX : "CENDRILLON" (PERRAULT) \\ ET «LES SIX CYGNES» (GRIMM)}

\section{Introduction}

$L$

ES INTERDITS PROLIFÈRENT DANS LES CONTES MERVEILLEUX. Certes, pour des récits aussi courts, ils génèrent une tension narrative avec une grande économie. Mais cet effet dramatique est-il leur seule fonction? Propp proposait déjà que la paire de fonctions interdiction/ transgression soit au fondement de la dynamique narrative. Il est vrai que dans certains contes la transgression d'un interdit posé par un personnage antagoniste, justifié par des motifs apparemment cohérents, est essentielle au récit (que l'on pense à «La Barbe bleue» de Perrault). Mais il apparaît que les situations d'interdits sont plus diverses que cela. Ainsi, a contrario, certains interdits posés par des personnages a priori bienveillants envers le héros n'entraînent pas forcément de transgression, et semblent anecdotiques ou à tout le moins peu justifiés dans le récit. Pour établir la fonction de ces interdits, nous allons analyser leur fonctionnement de manière minutieuse, en distinguant la règle elle-même, de ses éventuels motif ${ }^{\mathrm{I}}$ et sanction. À travers deux cas, "Cendrillon» et "Les six cygnes"", étudiés d'abord distinctement puis analysés selon leurs similarités, nous envisagerons la possibilité que l'interdit puisse parfois être salutaire.

I. J'utiliserai dans cet article le terme de "motif» au sens usuel et juridique de raison d'agir, et non au sens narratologique d'élément constituant du conte.

2. Le titre complet du conte est "Cendrillon ou la petite pantoufle de verre» de Perrault (I697), que nous indiquerons simplement par le premier mot, mais entre guillemets pour désigner le récit, et ainsi le distinguer du personnage; Die sechs Schwäne (KHM 49) des Grimm (I857).

Féeries, $\mathrm{n}^{\circ} \mathrm{1} 3,2016$, p. 87-115.

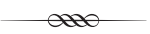




\section{Le cas Cendrillon : l'interdit de minuit}

Dans ce conte, rappelons qu'une jeune fille en haillons, opprimée par sa famille, souhaite en vain aller au bal; sa marraine bienveillante lui procure par magie équipage et tenue magnifiques mais la prévient qu' elle doit rentrer à minuit, au risque de reprendre son apparence initiale.

Voici le passage de l'interdit de minuit :

Quand elle fut ainsi parée, elle monta en carrosse; mais sa Marraine lui recommanda sur toutes choses de ne pas passer minuit, l'avertissant que si elle demeurait au Bal un moment davantage, son carrosse redeviendrait citrouille, ses chevaux des souris, ses laquais des lézards, et que ses vieux habits reprendraient leur première forme. Elle promit à sa Marraine qu'elle ne manquerait pas de sortir du Bal avant minuit. Elle part, ne se sentant pas de joie.

Examinons cette scène. La marraine annonce à sa filleule cette règle de couvre-feu in extremis ("monta en carrosse»), après un long passage merveilleux de métamorphose en princesse (d'un état cendreux à un état étincelant). Elle lui en souligne d'emblée l'importance («sur toutes choses») et formule la règle de manière précise («ne pas passer minuit» donc le temps des douze coups eux-mêmes est sauf, comme le deuxième soir le prouvera). Remarquons que, bien que l'interdit soit formulé positivement, presque comme un conseil, cette recommandation est assortie d'un avertissement, véritable sanction en cas de transgression.

\section{L'interdit non motivé}

Pourtant, la marraine n’indique pas de motif à cet interdit. Face à une règle aussi cruellement frustrante, pour qui a tant rêvé de ce bal, et en apparence arbitraire, ne cédons pas à la tentation de n'y trouver qu'une simple astuce pour créer de la tension narrative. Certains penseront qu'il s'agit d'une invraisemblance gratuite propre au genre du conte merveilleux; mais nous savons qu'au contraire la part de merveilleux est aussi limitée que possible dans le genre spécifique des contes-de-Perrault ${ }^{3}$, et que ces récits font preuve d'une cohérence extrême ${ }^{4}$. Certains esprits moralistes jugent que la capacité magique temporellement limitée représente le légitime prix à

3. Comme l'ont montré notamment Jean-Paul Sermain (2005), Marc Escola (2005) et JeanMichel Adam (2010), l'ensemble des récits des recueils de fiction de Perrault de I694, I695 et I697 forment un corpus d'une grande cohérence générique, comprenant des récits qualifiés aussi bien de contes que d'histoire ou nouvelle, des textes en vers et en prose.

4. Cette position ne peut être argumentée dans le cadre de cet article, mais nous espérons qu'il contribuera précisément à montrer par l'exemple qu'un sens cohérent est accessible. 
payer pour le don que reçoit la jeune fille, sur le mode d'un on-ne-peutpas-tout-avoir. Mais, comme nous le verrons, si morale il y a, c'est une leçon de vie que nous offre ce conte.

D'autres considéreront que la marraine ne fait qu'énoncer une condition technique indépendante de sa volontés, une sorte de contrainte de nature magique. Certes, le silence de la marraine à ce sujet pourrait le laisser accroire (et peut-être à dessein, comme nous le verrons plus loin). Mais, s'il est vrai que dans le monde des contes-de-Perrault les fées ne disposent pas de pouvoirs illimités, quand il s'agit d'incapacité féerique, elles le disent clairement : "Je n'ai pas assez de puissance pour défaire entièrement ce que mon ancienne a fait» dit la bonne fée de "La Belle au bois dormant»; "Je ne puis rien pour elle, Madame, du côté de l'esprit, lui dit la Fée, mais je puis tout du côté de la beauté» dans "Riquet à la houppe». Ce n'est donc pas le cas ici. D’ailleurs la fin du récit le prouvera puisque, une fois Cendrillon reconnue comme la jeune fille du bal, «la Marraine, [...] ayant donné un coup de sa baguette sur les habits de Cendrillon, les fit devenir encore plus magnifiques que tous les autres. [...] On la mena chez le jeune Prince, parée comme elle était». Ainsi, quand elle le veut, la fée peut transformer les vêtements de manière perpétuelle sans énoncer

5. C'est le motif utilisé dans le film d'animation Cinderella (1950) de Walt Disney et dans la comédie musicale télévisée Cinderella (I997) de Robert Iscove, dont voici respectivement les ultimes échanges entre la marraine et Cinderella :

"-Yes, my child, but like all dreams, well, I'm afraid this can't last forever. You'll have only 'tiI midnight and then... I - Midnight? Oh, thank you. / _ Oh, now, now just a minute. You must understand, my dear. On the stroke of twelve, the spell will be broken, and everything will be as it was before. I - Oh, I understand, but... it's more than I ever hoped for. I -Bless you, my child.

- There's one tiny little thing before I forget. You must leave while before the clock strikes twelve. It's very important. I - Before twelve? I - Look I don't make the rules. The magic only lasts till midnight. Come on Cinderella it's time to go.»

Henri Pourrat publie le conte Marie-Cendron dans Le Trésor des contes en 1948. Ici, c'est à la messe et non au bal qu'est empêchée de se rendre la jeune fille :

"Ainsi [somptueusement] mise, la fée l'envoya à l'église. Mais ce fut en lui recommandant de sortir avant le dernier évangile. - Peut-être que l'habillement était fée? Peut-être que l'enchantement ne devait tenir que juste pour ce temps de la messe? Toujours est-il que si la Cendroune n'était pas sortie au juste moment, il lui arriverait malencontre." (H. Pourrat, Le Trésor des contes, 1948, p. 56) (Je souligne) Ainsi, de façon plus sophistiquée, le narrateur pose de manière rhétorique la question du motif mais pour mieux l'esquiver en laissant croire que là n'est pas l'enjeu (nous allons plus loin que Jean-Michel Adam («Le dialogue intertextuel du Trésor des Contes d'Henri Pourrat avec les Grimm et Perrault», Féeries, n 9, 2012, p. I80) sur ce point : "“Toujours est-il que" laisse entendre que ce qui suit est narrativement seul digne d'intérêt et que, rétroactivement, l'état de choses auquel la locution renvoie doit être considéré comme une question secondaire.»). 
de contrainte. Reconnaissons donc que l'interdit posé par la marraine est délibéré et par elle fixé.

S'agit-il, comme l'affirme Joseph Courtès ${ }^{6}$, d'un "échange» que propose la marraine : elle octroierait un don et s'attendrait en retour à un contre-don d' "obéissance»? Cela est d'abord absurde, et assimilerait la marraine à un tyran qui, pour prix d'une aide supposément motivée par l'affection, demanderait une obéissance à un ordre gratuit. Ensuite, cela serait étonnant, et sans doute un peu malhonnête, de formuler cette demande in extremis, et d'ailleurs la marraine a déjà demandé initialement, de manière plus appropriée, comme condition, d'exécuter diligemment ses instructions préparatoires : «Tu voudrais bien aller au Bal, n'est-ce pas? [...] seras-tu bonne fille? dit sa Marraine, je t'y ferai aller.»

S'agit-il d'un couvre-feu de bienséance? Étrange bienséance qui ne concernerait que Cendrillon et ni ses deux sœurs, pourtant "demoiselles", ni les autres jeunes filles invitées, pourtant "personnes de qualité» («le fils du Roi donna un bal, et [...] il en pria toutes les personnes de qualité : nos deux Demoiselles en furent aussi priées»). D’ailleurs Jean-François Solnon explique qu'à la Cour, à Versailles, «le bal [...] se prolonge jusqu’au matin ${ }^{7}$ ».

$S^{\prime}$ agit-il d'une réaction quasi maternelle excessivement protectrice? C’est ce qu'affirme Bruno Bettelheim : «L'ordre de la fée [...] fait penser aux parents qui ne veulent pas que leur fille s'attarde le soir hors de la maison, de peur qu'il ne lui arrive des choses épouvantables ${ }^{8}$.» Il est certainement vrai que les parents imaginent le doux foyer familial comme plus protecteur qu'une sortie de leur enfant, encore immature, dans un lieu inconnu d'eux et peut-être malfamé, avec des compagnons inconnus d'eux dont les parents sont inconnus d'eux. Mais étrange protection, en ce qui concerne le récit de Perrault, qui voudrait que la jeune fille si perspicace (et en âge de se marier) qu' est Cendrillon, maltraitée à la maison, y soit plus en sécurité qu'en public à la Cour royale, se faisant passer pour une princesse et accompagnée de sept serviteurs (six laquais et un cocher). D'ailleurs, l'attitude de la marraine ne correspond en rien à une telle inquiétude, à la fois dans la formulation initiale de sa "recommandation» mais aussi en ne répétant pas sa mise en garde pour le deuxième soir (Cendrillon «alla trouver sa Marraine et [...] elle lui dit qu'elle souhaiterait bien aller encore le lendemain au $\mathrm{Bal}$ »; sans provoquer de réaction), comme le ferait certainement un parent inquiet.

6. J. Courtès, Introduction à la sémiotique narrative et discursive, 1980, p. I25-I26.

7. J.-Fr. Solnon, La Cour de France, 1987, p. 455.

8. Br. Bettelheim, Psychanalyse des contes de fées, 2007, p. 392-393. 
Au contraire, nous allons montrer que la marraine, qui est une figure maternelle expérimentée et non abusive ${ }^{9}$, donne sa chance à sa filleule de gagner son autonomie de la manière la plus habile. C'est d'ailleurs ce que nous dit Perrault dans la première moralité («sa Marraine, en la dressant $^{\mathrm{I}}{ }^{\mathrm{O}}$, en l'instruisant, [...] en fit une Reine»). Car en cet interdit délibéré (mais au motif caché) réside l'essence (cachée) de ce conte-ci. À la suite de Bernadette Bricout ${ }^{\text {II }}$, j'interprète la règle de minuit comme un enseignement sur l'art du désir et de la séduction.

Envisageons en effet la situation. La jeune fille se rend à un bal, «lieu de la rencontre, [qui] permet, par le biais de l'invitation et de la danse qui la suit, le rapprochement des corps, l'harmonisation des mouvements et les conversations privées au sein même de l'espace public ${ }^{12} »$. Au bal, on se frôle et on se murmure à l'oreille pour mieux se séduire dans un érotisme diffus. En s'échappant soudainement, Cendrillon frustre le prince (d'autant plus qu'il doit lui sembler extraordinaire qu'une femme puisse lui résister) et attise ainsi son désir ${ }^{13}$.

9. Dans le monde des contes-de-Perrault, les fées-marraines ne sont pas seulement dotées de capacités magiques ou de bienveillance quasi-maternelle, elles font preuve aussi de bon sens psychologique :

- dans "Peau d'Âne» : "Je sais, dit-elle, en voyant la Princesse, Ce qui vous fait venir ici, Je sais de votre cœur la profonde tristesse; Mais avec moi n'ayez plus de souci. Il n'est rien qui vous puisse nuire Pourvu qu’à mes conseils vous vous laissiez conduire»; «la sage fée»;

- dans «La Belle au bois dormant» : «[...] grandement prévoyante, elle pensa que quand la Princesse viendrait à se réveiller, elle serait bien embarrassée toute seule dans ce vieux Château. »;

- dans «Riquet à la houppe» : "Ne vous affligez point tant, Madame, lui dit la Fée; votre fille sera récompensée d'ailleurs, et elle aura tant d'esprit, qu'on ne s'apercevra presque pas qu'il lui manque de la beauté.»

Io. Selon le Dictionnaire universel de Furetière (1690), «dresser» signifie "instruire et disposer à faire quelque chose».

II. B. Bricout, La clé des contes, 2005, p. I40.

I2. Ibid., p. I38.

13. Cette idée est d'ailleurs un topos de la littérature classique. Perrault lui-même, dès I660, écrit un Dialogue de l'amour et de l'amitié. Dans sa Lettre à Monsieur l'abbé d'Aubignac qui l'accompagne, il établit déjà ce principe de séduction : «L'Amour [...] se port[e] avec plus d'empressement et de chaleur aux choses qui lui sont défendues, qu'à celles qui lui sont permises.» (Ch. Perrault, Contes, 1967, p. 2II) En 1665, dans ses Réflexions ou sentences et maximes morales, François de La Rochefoucauld aphorise que "n'aimer guère en amour est un moyen assuré pour être aimé». En I669, dans Les Amours de Psyché et de Cupidon de La Fontaine, comme me l'a soufflé Jean-Paul Sermain, le dieu de l'amour professe que "du moment que vous n'aurez plus rien à souhaiter, vous vous ennuierez. [...] le meilleur pour vous est l'incertitude, et qu'après la possession vous ayez toujours de quoi désirer" (Fr. Charpentier [éd.], I990, p. 72). Puis, plus tard, la "coquetterie» féminine consistant à se dérober pour se faire désirer deviendra, selon René Girard (Mensonge romantique et vérité romanesque, 200I, p. I38) qui s'efforce d'en expliquer le mécanisme, un thème majeur, notamment chez Marivaux. 
Bien que la raison de l'interdit ne lui soit pas donnée explicitement, Cendrillon, qui est d'une grande finesse psychologique ${ }^{\mathrm{I}}$, construit exprès son mystère. En premier lieu, elle conserve son incognito : " [...] il venait d'arriver une grande Princesse qu'on ne connaissait point [...]. Il se fit alors un grand silence [...] tant on était attentif à contempler les grandes beautés de cette inconnue. [...] Cendrillon [...] demanda [à ses sœurs] le nom de cette Princesse; mais elles lui répondirent qu'on ne la connaissait pas, que le Fils du Roi en était fort en peine, et qu'il donnerait toutes choses au monde pour savoir qui elle était. Cendrillon sourit [...].» Dirat-on qu' elle agit ainsi pour la simple raison qu'elle ne peut en aucun cas révéler, à ce stade, son identité réelle? Certes, mais elle aurait pu s'inventer une identité imaginaire, comme le décide le Chat botté, dans le monde-de-Perrault, en affublant son maître du titre ronflant aux sonorités exotiques de marquis de Carabas. Cendrillon et le Chat mettent bien en œuvre deux procédés identitaires opposés, adaptés à leurs stratégies respectives. Cendrillon, en se refusant à s'identifier, intrigue la compagnie et elle le sait (et en «sourit») Is.

En second lieu, donc, se soumettant à la consigne reçue, Cendrillon disparaît ${ }^{16}$. De fait, elle en apprécie dès le premier soir le résultat positif, en termes de séduction, lorsqu'elle se fait rapporter par ses sœurs le récit du bal.

Puis, en troisième lieu, le deuxième soir, en laissant tomber une pantoufle, ce n'est pas seulement un indice pour être retrouvée qu'elle sème, mais aussi un gage fétichiste (puisqu'il n'a rien d'autre, pas même son nom, auquel se raccrocher) qu' elle laisse à son prince-charmé pour cristal-

I4. Nous en avons de multiples témoignages dans le récit : elle pleure pour émouvoir sa marraine et lui faire exprimer sa demande à sa place, elle l'interroge de manière rhétorique, elle manipule ses sœurs, etc.

15. Le conte apparenté Datte, belle datte de Italo Calvino (Contes populaires italiens, 1984, p. 13) illustre plaisamment ce mystère qui excite la curiosité :

"Onze heures trois quart sonnèrent à l'horloge. / - Monseigneur, je suis obligée de m'en aller! / —Déjà, le bal n'est pas encore fini! / —Oui mais moi, j'ai promis de rentrer, de rentrer à cette heure-là. Vous savez, je peux être punie. / - Je n'aimerais pas que vous soyez punie. Allez-vous revenir? / —Oui, je reviendrai. / _Comment vous appelez-vous? / — Par mon nom. / -Et où logez-vous? / —Dans la maison qui a une porte. / _Dans quelle rue? / —Dans la ruelle où il y a de la poussière. / —Madame, vous me faites mourir. / — À votre aise!»

Mais Cendrillon, elle, ne garantit même pas au prince qu'elle reviendra!

I6. Notons que l'heure imposée, minuit, devient compréhensible à partir des principes d'incognito et de disparition que met en œuvre Cendrillon. Les bals d'apparat se terminent souvent vers une heure du matin. C'est pourquoi le choix de minuit est un bon pronostic de la marraine pour limiter le risque que sa filleule ne s'attarde au-delà de la fin du bal, où il lui serait alors difficile de s'éclipser facilement. 
liser son désir : «Le fils du Roi [...] avait ramassée [la pantoufle], et [...] n'avait fait que la regarder pendant tout le reste du $\mathrm{Bal}^{17}$."

Tentons un raisonnement par l'absurde de nature non plus argumentative mais narrative. Ainsi, imaginons ce qui se passerait si aucune consigne n’avait été donnée à Cendrillon : exultant de pouvoir enfin passer une nuit à un bal princier, Cendrillon guettait tout de même d'un œil ses sœurs afin de s'assurer seulement de les précéder au moment où celles-ci se décideraient à rentrer, perdant ce faisant un peu de son naturel. Le prince, à force de discussions avec elle, finit par s'étonner de ses réponses étranges pour une supposée princesse, s'entêtant dans son incognito, puis ils n'eurent plus rien à se dire ${ }^{18}$. Enfin, au petit matin, lorsque toute la compagnie se sépara tranquillement, encore intéressée par elle, le prince la fit accompagner (d'autres disent qu'il l'a suivie) : son charmant secret fut éventé, le prince en fut tout déconfit.

$\mathrm{Au}$ contraire de ce récit alternatif aboutissant à une impasse, dans la logique du conte, grâce à l'interdit salutaire, Cendrillon sait désormais, en jouant de son mystère, de ses apparitions et disparitions en s'esquivant par deux fois, de sa présence-absence imprimée en négatif dans un moulage translucide ${ }^{19}$, entretenir le charme : elle maîtrise l'art de séduire ${ }^{20}$.

\section{Une moralité sibylline}

Notre interprétation est-elle conforme à celle que propose l'auteur luimême dans ses moralités? Perrault fait suivre le récit de deux moralités. La première s'adresse aux femmes et évoque les qualités féminines nécessaires au succès conjugal ${ }^{21}$ :

17. La réaction du prince du conte apparenté «Finette Cendron» de Madame d'Aulnoy, en I698, est encore plus claire : «Il la fait ramasser, la regarde, en admire la petitesse et la gentillesse, la tourne, retourne, la baise, la chérit et l'emporte avec lui.»

I8 Dans le monde-de-Perrault, le conte "La Belle au bois dormant» établit implicitement à un peu plus de huit heures (soit une longue nuit de bal) la durée avant que la première conversation ne se tarisse entre deux inconnus frappés réciproquement de coup de foudre amoureux : «Il y avait quatre heures qu'ils se parlaient, et ils ne s'étaient pas encore dit la moitié des choses qu'ils avaient à se dire.» Or, dans le récit qui nous occupe, l'attachement n'étant pas initialement aussi réciproque, il s'ensuit que la conversation durerait moins longtemps.

I9 Le potentiel érotique de telles pantoufles est, semble-t-il, apprécié par les strip-teaseuses de pole dance qui portent des escarpins en plexiglas.

20 Finalement Joseph Courtès ne dit pas autrement avec son vocabulaire sémiotique : «La fuite de Cendrillon (et [...] son refus de dire qui elle est) [...] la fait passer [de] l'état mensonger [à] l'état de secret [comme] faire-persuasif.» (ouvr. cité, p. 134-I35)

2I Et la seconde moralité lui réplique, pertinemment mais hors de propos pour nous ici, en arguant, avec réalisme voire cynisme, que pour obtenir le succès social (au-delà du seul conjugal), toutes les qualités que l'on puisse posséder sont insuffisantes sans relations influentes — comme l'illustre le récit. 
I La beauté $[\ldots]$ est un rare trésor,

3 [...] Mais ce qu'on nomme bonne grâce 22

$4[\ldots]$ vaut mieux encor.

5 C'est ce qu'à Cendrillon fit avoir sa Marraine,

6 En la dressant, en l'instruisant,

7 Tant et si bien qu'elle en fit une Reine :

$9[\ldots]$ ce don vaut mieux que d'être bien coiffées,

Io Pour engager un cœur, pour en venir à bout,

II La bonne grâce est le vrai don des Fées;

I2 Sans elle on ne peut rien, avec elle, on peut tout.

Qu'est-ce donc ce que Perrault nomme «bonne grâce», qui, plus que la beauté naturelle (v. I) ou l'apprêtement artificiel (v. 9), emporte le coeur (v. Io) ? On pourrait croire naïvement qu'il s'agit de la gentillesse, de la bonté et autres qualités morales que Perrault loue par ailleurs et dont il dote d'emblée Cendrillon : "[...] une jeune fille, [...] d'une douceur et d'une bonté sans exemple." Non, car cette bonne grâce ne produit pas simplement un caractère agréable à vivre ${ }^{23}$ mais est, selon la moralité, une qualité essentielle à la relation amoureuse. La bonne grâce est-elle simplement une grâce bonne ${ }^{24}$, une grâce supérieure? Non, car le monde-dePerrault distingue bien ces deux qualités : ainsi dans «La Belle au bois dormant", la princesse reçoit en dot "qu' elle aurait une grâce admirable à tout ce qu'elle ferait»; ainsi dans la nouvelle «La Marquise de Salusses ou la Patience de Griselidis", l'héroïne fait preuve, en rencontrant son futur amant, de «joie» et de «bonne grâce» qui le séduisent aussitôt, tandis que les courtisanes au contraire «diligemment / Mettent tout leur savoir et toute leur adresse / À donner de la grâce à cha[cun de leur] ajustement». La grâce est certes un charme particulier, mais la bonne grâce, plus guère utilisée dans le français contemporain que dans la locution adverbiale «de bonne grâce", est définie encore au XIX ${ }^{\mathrm{e}}$ siècle comme une "grâce relevée de quelque chose de simple, de franc et de libre ${ }^{25}$ ». Dans les traités de civilité du XVII ${ }^{\mathrm{e}}$ siècle, ce trait de caractère est considéré comme l'un des meilleurs atouts dans l'art de plaire. Du Bosc, dans son ouvrage L'honneste femme de 1632, lui consacre un chapitre entier. La bonne grâce se manifeste par une grâce non pas affectée mais naturelle, joyeuse, vive, instinctive,

22. Je souligne.

23. Dans le monde-de-Perrault, comme l'illustre bien le conte «Les Fées», s'oppose en effet les «humeur[s]» empreintes de "douceur» et d' «honnêteté» à celles «si désagréables et si orgueilleuses qu'on ne pouvait vivre avec elles».

24. On sait l'impact au niveau du sens, en français, dans la syntaxe des adjectifs épithètes, de l'antéposition ou de la postposition.

25. É. Littré, Dictionnaire de la langue française (I872-77), «GRÂCE». 
un peu imparfaite, presque négligente ${ }^{26}$. Le don précieux de la marraine à Cendrillon, serait-ce ce côté un peu fantasque de la jeune fille qui, d'une généreuse révérence et sans la moindre explication, se volatilise?

Le temps d'une soirée, Cendrillon réussit à charmer le prince. Examinons-en les étapes. Lors de son arrivée, elle éblouit le prince et toute la compagnie par sa beauté resplendissante. Dans un second temps, «elle dansa avec tant de grâce, qu'on l'admira encore davantage». Sa grâce fait son effet mais il ne s'agit encore que d'admiration collective même si déjà le cour du prince est bien engagée (voir v. Io) et il ne la quitte plus des yeux, observant avec quel naturel elle partage avec deux apparemment quelconques demoiselles les fruits luxueux qu'il lui avait donnés. Enfin, elle vient à bout du cœur du prince quand, comble de la bonne grâce, elle repart aussi mystérieusement et galamment qu'elle était apparue.

La moralité précise que cette «bonne grâce» est le résultat d'un apprentissage dû à la marraine (v. 6). Or, le seul impact, à travers le récit, de la marraine dans le comportement de Cendrillon consiste dans son départ précipité du bal ${ }^{27}$. Il s'ensuit donc bien que la bonne grâce en question dans la moralité soit l'enseignement sur l'art de séduire que procure l'interdit de minuit. D’ailleurs y est distingué le "vrai don" (v. II), qu'est la bonne grâce, du don moins "vrai», au sens de son caractère bénéfique, qu'est la parure que Cendrillon reçoit.

Mais ce vers (II) recèle néanmoins un sens équivoque, car les "fées» $y$ sont étrangement plurielles ${ }^{28}$, convoquant de manière subtile la scène primaire où des fées prodiguent des dons au nouveau-né ${ }^{29}$. Comme si la moralité proclamait certes le caractère acquis de la bonne grâce (v. 6) mais aussi, de manière quelque peu sibylline, son caractère inné (v. II). Parallèlement, les traités de civilité traitent de la bonne grâce avec la même ambiguïté : d'une part une attitude savamment négligente, subtilement oxymorique $^{30}$, et fruit d'une éducation; d'autre part une disposition natu-

26. J. Du Bosc, L'Honnête femme, I665, p. I45.

27. Lorsque sa marraine demande d'entrée de jeu à Cendrillon d'être «bonne fille», il s'agit d'un échange, puisque c'est une condition qu'elle pose à sa coopération, et non d'une injonction éducative. En outre «bonne fille» recouvre un sens particulier dans le monde-de-Perrault, mais nous en reparlerons plus loin.

28. Comme le titre du conte «Les Fées» abondamment discuté par la critique (notamment Soriano).

29. Voir «La Belle au bois dormant» et «Riquet à la houppe».

30. Remarquons qu'on peut se demander s'il n'est pas fait allusion à la nature oxymorique de la situation de Cendrillon dès le titre du récit : Cendrillon ou la petite pantoufle de verre. En effet, la pantoufle est une chaussure souple et légère, tandis que le verre est un matériau cassant et relativement lourd. Il faut bien posséder la bonne grâce de Cendrillon pour parvenir à la porter pour 
relle et spontanée. Ainsi, «la bonne grâce se remarque à faire tout comme par nature et sans étude ${ }^{31}$ " qu'il faut "cacher subtilement, sans qu'on découvre l'artifice ${ }^{32}$; " user partout d'une certaine négligence qui cache l'artifice et témoigne que l'on ne fait rien que comme sans y penser, et sans aucune sorte de peine ${ }^{33}$ ».

Comment résoudre ce paradoxe d'une grâce acquise qui doit apparaître comme un don naturel? D'ailleurs, s'agissant de Cendrillon, il semble bien qu'à la fois elle bénéficie d'un talent naturel et qu'elle a finement intégré l'enseignement de sa marraine... Précisément, nous verrons plus loin comment l'interdit participe à la résolution de ce problème.

\section{Cendrillon versus Aschenputtel : interdire ou pas}

À ce stade, il est frappant de remarquer que le conte apparenté ${ }^{34}$, "Aschenputtel» des Grimm, ne contient pas d'interdit : là, la jeune femme, nommée Aschenputtel, quitte également le bal de manière précoce (voir les lignes IO-I2 du tableau $\mathrm{n}^{\circ} \mathrm{I}$ ) mais sans qu'aucune consigne extérieure ne l'y contraigne. En effet, elle le décide de sa propre volonté (voir 1. 13-15), qui est nettement répétée sans être a priori justifiée.

Est-ce à dire que l'interdit n'est pas nécessaire à ces histoires? En fait, si on examine de près les deux récits, il s'agit d'histoires bien différentes. Comment expliquer qu'Aschenputtel veuille ainsi rentrer hâtivement en dépit de la tentation si compréhensible de vouloir faire durer le délicieux moment du bal? Je montrerai qu'elle est mue non par le désir de séduire, mais par la crainte de sa famille.

Pour le comprendre, il faut procéder à une comparaison analytique des deux récits $\left(\right.$ tableau $\left.{ }^{\circ} \mathrm{I}\right)$.

danser «avec tant de grâce» et courir «aussi légèrement qu'aurait fait une biche». Cet oxymore est délibérément recherché, sinon pourquoi ne pas employer le mot "soulier», ou même le mot " mule» (qui désigne une chaussure n'ayant pas autant la même caractéristique de souplesse) comme dans "Finette Cendron"? Et pourquoi rappeler le syntagme pantoufle de verre dans le titre alors que le surnom Cendrillon eût suffit, comme dans d'autres titres de contes-de-Perrault?

31. J. Du Bosc, ouvr. cité, p. I45.

32. Ibid., p. I46.

33. N. Faret, L'honnête homme ou l'art de plaire à la Cour, I630, p. 35.

34. Je ne qualifierai pas ces contes comme étant des versions d'un même conte-type, comme le fait la tradition folkloriste, ces notions me semblant problématiques, et je préfère les qualifier de contes apparentés formant une famille, un peu à la manière dont Wittgenstein parle d'air de famille; mais cela ne peut faire l'objet d'une argumentation dans le cadre du présent article. 
Tableau $\mathbf{n}^{\circ} \mathbf{I}$

\begin{tabular}{|c|c|c|c|c|c|}
\hline Récit & & & "Cendrillon" & «Aschenputtel» & $\mathrm{L}$ \\
\hline \multirow{8}{*}{$\begin{array}{l}\text { Relations } \\
\text { fami- } \\
\text { liales de } \\
\text { l'héroïne }\end{array}$} & \multirow[b]{3}{*}{$\begin{array}{l}\text { en } \\
\text { général }\end{array}$} & belle-mère & $\begin{array}{l}\text { Elle «chargea [l’héroïne] } \\
\text { des plus viles occupations } \\
\text { de la Maison» et la faisait } \\
\text { "couch[er] dans un grenier, } \\
\text { sur une méchante paillasse" }\end{array}$ & & 1 \\
\hline & & sœur aînée & & \multirow[b]{2}{*}{$\begin{array}{l}\text { «cœur }[\ldots] \text { méchant et } \\
\text { noir»; « Que fait cette pauvre } \\
\text { sotte }[\ldots . .] \text { avec nous? [...] } \\
\text { dehors la fille de cuisine!»; } \\
\text { «elles ôtèrent }[\ldots . .] \text { ses beaux } \\
\text { habits»; « Voyez un peu } \\
\text { comme cette princesse si fière } \\
\text { est endimanchée! s'excla- } \\
\text { mèrent-elles en pouffant de } \\
\text { rire»; elles «lui infligeaient } \\
\text { toutes les misères imagi- } \\
\text { nables, elles se moquaient } \\
\text { d’elle et lui renversaient ses } \\
\text { pois et ses lentilles dans les } \\
\text { cendres» }\end{array}$} & 2 \\
\hline & & $\begin{array}{l}\text { sœur } \\
\text { cadette }\end{array}$ & $\begin{array}{l}\text { Elle «n’était pas si malhon- } \\
\text { nête que son aînée, [et] } \\
\text { l'appelait Cendrillon» }\end{array}$ & & 3 \\
\hline & \multirow{5}{*}{ le bal } & belle-mère & & $\begin{array}{l}\text { Autorise l'héroïne à aller au } \\
\text { bal contre une corvée puis } \\
\text { viole deux fois sa promesse }\end{array}$ & 4 \\
\hline & & préparation & $\begin{array}{l}\text { "[les sœurs] appelèrent Cen- } \\
\text { drillon pour lui demander } \\
\text { son avis, car elle avait le goût } \\
\text { bon. Cendrillon les conseilla } \\
\text { le mieux du monde, et s'offrit } \\
\text { même à les coiffer; ce qu'elles } \\
\text { voulurent bien " }\end{array}$ & $\begin{array}{c}\text { L'héroïne est requise par ses } \\
\text { sœurs de les préparer pour } \\
\text { le bal et elle «obéit mais en } \\
\text { pleurant» }\end{array}$ & 5 \\
\hline & & au bal & $\begin{array}{l}\text { «Elle alla s'asseoir auprès de } \\
\text { ses sœeurs, et leur fit mille } \\
\text { honnêtetés : elle leur fit part } \\
\text { des oranges et des citrons que } \\
\text { le Prince lui avait donnés» }\end{array}$ & I & 6 \\
\hline & & $\begin{array}{l}\text { à l'issue du } \\
\text { bal }\end{array}$ & $\begin{array}{l}\text { Les sœurs discutent avec } \\
\text { l'héroïne } 2 \text { fois }\end{array}$ & l & 7 \\
\hline & & $\begin{array}{l}\text { dénoue- } \\
\text { ment }\end{array}$ & $\begin{array}{l}\text { Les sœurs «se jetèrent à ses } \\
\text { pieds pour lui demander } \\
\text { pardon [...]. Cendrillon } \\
\text { les releva, et leur dit, en } \\
\text { les embrassant, qu'elle leur } \\
\text { pardonnait de bon cœur, et } \\
\text { qu'elle les priait de l'aimer } \\
\text { bien toujours [et les] fit loger } \\
\text { [...] au Palais, et les maria } \\
\text { dès le jour même à deux } \\
\text { grands Seigneurs de la Cour» }\end{array}$ & $\begin{array}{l}\text { Belle-mère et sœurs "pâlirent } \\
\text { de colère» puis «perfides } \\
\text { [tentent] de s'insinuer dans } \\
\text { ses bonnes grâces [mais] les } \\
\text { colombes leur crevèrent alors } \\
\text { un œil [puis] l'autre" }\end{array}$ & 8 \\
\hline
\end{tabular}




\begin{tabular}{|c|c|c|c|c|c|}
\hline Récit & & & "Cendrillon" & «Aschenputtel» & $\mathrm{L}$ \\
\hline \multirow{10}{*}{ Le bal } & $\begin{array}{l}\text { intention } \\
\text { conjugale }\end{array}$ & & l & $\begin{array}{l}\text { "pour que [le prince] puisse } \\
\text { choisir une fiancée» }\end{array}$ & 9 \\
\hline & \multirow{3}{*}{ départ } & soir 1 & "onze heures trois quarts" & \multirow{3}{*}{ Le soir venu } & 10 \\
\hline & & soir 2 & «Minuit» & & 11 \\
\hline & & soir 3 & l & & 13 \\
\hline & \multirow{3}{*}{ raison } & soir 1 & \multirow{2}{*}{$\begin{array}{l}\text { l'héroïne part comme } \\
\text { «recommandé» }\end{array}$} & L'héroïne «voulut rentrer» & 14 \\
\hline & & soir 2 & & L'héroïne «voulut partir» & 15 \\
\hline & & soir 3 & 1 & L'héroöne "voulut s'en aller" & 16 \\
\hline & \multirow{3}{*}{ attitude } & soir 1 & $\begin{array}{c}\text { "fit aussitôt une grande } \\
\text { révérence à la compagnie, et } \\
\text { s'en alla" }\end{array}$ & $\begin{array}{l}\text { "[s] échappa }[\ldots] \text { et se réfu- } \\
\text { gia dans le pigeonnier» }\end{array}$ & 17 \\
\hline & & soir 2 & $\begin{array}{l}\text { "s'enfuit aussi légèrement } \\
\text { qu'aurait fait une biche» }\end{array}$ & «se sauva» & 18 \\
\hline & & soir 3 & 1 & "[s']échappa» & 19 \\
\hline \multirow[t]{4}{*}{ L'essayage } & & agent & Un "gentilhomme» & Le prince & 20 \\
\hline & & $\begin{array}{l}\text { initiative de } \\
\text { l'héroïne }\end{array}$ & $\begin{array}{l}\text { "Cendrillon qui les regardait } \\
\text { [...] dit en riant: "Que je } \\
\text { voie si elle ne me serait pas } \\
\text { bonne!" Ses sœurs se mirent } \\
\text { à rire et à se moquer d'elle. } \\
\text { Le Gentilhomme [...] [la] fit } \\
\text { asseoir [...]" }\end{array}$ & $\begin{array}{l}\text { L'héroïne par trois fois ne } \\
\text { tente pas de participer à } \\
\text { l'essayage; la belle-mère tente } \\
\text { de l'empêcher : "Le fils du } \\
\text { roi lui dit de la faire monter, } \\
\text { mais la mère répliqua : "Oh } \\
\text { non ! [...]"” }\end{array}$ & 21 \\
\hline & & $\begin{array}{l}\text { la preuve } \\
\text { ultime }\end{array}$ & $\begin{array}{l}\text { L'héroïne «tira de sa poche } \\
\text { l'autre petite pantoufle» }\end{array}$ & \multirow[b]{2}{*}{$\begin{array}{l}\text { Le prince "posa les yeux sur } \\
\text { son visage [et la] reconnut" }\end{array}$} & 22 \\
\hline & & $\begin{array}{l}\text { retrouvailles } \\
\text { princières }\end{array}$ & $\begin{array}{l}\text { «On la mena chez le jeune } \\
\text { Prince, parée [encore plus } \\
\text { magnifiquement] : il la } \\
\text { trouva encore plus belle que } \\
\text { jamais, et peu de jours après, } \\
\text { il l'épousa» }\end{array}$ & & 23 \\
\hline
\end{tabular}

Bettelheim propose une explication psychologique: Aschenputtel «montre qu'elle veut être choisie pour ce qu'elle est vraiment et non pour ses atours somptueux. Elle n'appartiendra à son amant que si, l'ayant vue dans son état de dégradation, il n'en continue pas moins de la désirer ${ }^{35}$ ", puis «elle refuse d'être choisie en raison d'une apparence due à la magie et $s^{\prime}$ arrange pour que le prince la découvre vêtue de ses haillons ${ }^{36}{ }^{3}$. Cet argument mérite d'être examiné rigoureusement. L'intention quasiment romantique que Bettelheim prête à l'héroïne est contredite par les faits

35. Br. Bettelheim, ouvr. cité, p. 391.

36. Ibid., p. 396. 
textuels: Aschenputtel n'essaye en aucune manière de se révéler au prince dans son état misérable. De fait, elle ne laisse aucun indice pour que le prince puisse la retrouver hors du bal, contrairement à Cendrillon (l. 19) ${ }^{37}$; elle ne s'efforce aucunement de participer aux essayages contrairement à Cendrillon (1. 2I); elle ne produit pas de sa poche le deuxième soulier au moment décisif contrairement à Cendrillon (1. 22); bref, Aschenputtel n'y est pour rien dans ses retrouvailles avec le prince, au contraire de Cendrillon.

En fait, la fuite d'Aschenputtel s'explique simplement si l'on compare finement les relations familiales des deux héroïnes.

Par rapport à celles de Cendrillon, les relations d'Aschenputtel avec ses belles-mères et demi-sœurs sont infiniment plus exécrables. Si Cendrillon est brimée et maltraitée, Aschenputtel est opprimée et martyrisée avec sadisme (l. I-4) $)^{38}$. L'une des sœurs de Cendrillon est plus «honnête» avec elle et c'est d'ailleurs elle qui lui donne le sobriquet ${ }^{39}$ le plus affectueux qu'utilise donc le narrateur; tandis que les trois antagonistes d'Aschenputtel sont indistinctement perverses ${ }^{40}$. Ses sœurs requièrent avec égards Cendrillon pour les préparer, tandis qu'Aschenputtel est sommée pour la même tâche et s'en acquitte avec réticence (1. 5). Cendrillon est présente lors de l'essayage et peut y participer même si elle est moquée par ses sœurs; tandis qu'Aschenputtel est tenue absente de l'essayage que sa belle-mère tente d'empêcher (1. 2I). Ses sœurs discutent avec Cendrillon à leur retour du bal (1. 7), et Cendrillon se les concilie durant le bal (1. 6) puis se réconcilie avec

37. Dans "Cendrillon", a minima subsiste une ambiguïté sur la nature accidentelle ou délibérée de son geste, tandis que c'est clairement le prince qui use d'un stratagème dans "Aschenputtel».

38. Dira-t-on que cela résulte du style des contes des Grimm qui serait en général plus cruel que celui des contes de Perrault? Non, car cela ne change rien à notre affaire : si nombre des situations des contes des Grimm sont certainement cruelles, celle d'Aschenputtel ne le serait pas moins pour autant. D'autre part, mon interprétation est renforcée par les faits co-textuels des personnages des contes-de-Perrault qui sont clairement cruels quand cela convient au récit, comme la belle-mère de "La Belle au bois dormant", et même la mère des "Fées". D’ailleurs, dans ce dernier cas, comme le remarque Jean-Michel Adam, la vérité contrastive des "Fées» avec "Cendrillon" est d'autant plus flagrante que "c'est manifestement l'ajout de Cendrillon, [dans le recueil de] 1697, qui explique les changements [dans le texte des] Fées de 1695 au point [...] d'introduire une cruauté maternelle pire que celle de la marâtre [de Cendrillon]» (Textualité et intertextualité des contes, 2010, p. 240). Cela signifie bien que la belle-mère de Cendrillon n'est pas si cruelle que cela!

39. Sobriquet dont «le suffixe "illon" n’est point péjorati[f]» (J. Courtès, ouvr. cité, p. II2).

40. Avec une évolution génétique cependant qui fait «circuler» l’antagonisme : «Les corvées pour l'empêcher d'aller [au bal] sont infligées par les sœurs [dans l'édition première de] I8I2 [...] alors que le rôle est repris par la marâtre [dans l'édition dernière de] I857." (C. François, "Échanges et dialogues intertextuels : l'exemple des contes de Perrault et des frères Grimm ", dans M. Bragança et S. Wilson (éds), É/change : transitions et transactions dans la littérature française / Ex/change: Transitions et Transactions in French Literature, 20II, p. 53) 
elles in fine (1. 8); tandis qu'Aschenputtel sera dûment vengée ${ }^{41}$ de leur cruauté et de leur perfidie (1. 8). Ainsi les deux jeunes femmes vivent des situations très différentes, et Aschenputtel s'avère essentiellement motivée par la peur.

Aussi, durant le bal, Cendrillon déploie une stratégie de séduction qui ne doit pas être mise en péril; tandis qu'Aschenputtel doit à tout prix éviter que ses demi-sœurs et sa marâtre ne la reconnaissent : d'un côté, une Cendrillon qui cache son identité y compris à ses sœurs pour qu'elles ne la divulguent pas et compromettent ainsi sa part de mystère; de l'autre, une Aschenputtel qui se méfie, à juste titre, du châtiment familial qu'elle subirait si sa présence au bal était découverte par ses sœurs (qui, de nature brutale ${ }^{42}$, sont prêtes à se mutiler pour épouser le prince). D'un côté, une Cendrillon qui quitte le bal légère et auréolée de son mystère; de l'autre, une Aschenputtel qui s'enfuit craintive (1. I6-I8). Notons enfin qu'Aschenputtel ne part pas vers «minuit» comme Cendrillon, soit un instant temporel précis approprié à un ordre précis, mais à chaque fois vaguement le «soir» (1. IO-I2), car elle doit anticiper le retour de ses sœurs. En effet, le départ précoce d'Aschenputtel répond à une autre logique que celle de Cendrillon, celle de la peur.

On pourrait s'étonner qu'Aschenputtel s'échappe du prince qui par trois fois la poursuit jusque dans sa maison. Ne devrait-elle pas, justement, se réfugier auprès de lui et s'en remettre à lui? Tout indique que la jeune femme éprouve une bien plus grande peur de ses sœurs et bellemère qu'elle n'a confiance, à ce stade, dans l'affection du prince pour la protéger.

Néanmoins, maintenant qu'est établie la motivation du comportement du personnage Aschenputtel, on peut faire la part des choses et comprendre en effet ${ }^{43}$ le message du conte «Aschenputtel» comme celui de la véritable rencontre amoureuse fondée sur la beauté intérieure et non sur les apparences. Mais, quoi qu'il en soit, ce n'est pas le message que propose

4I. Dans d'autres contes apparentés, les sœurs sont «seulement» transformées à leur tour en «domestiques» (J. Courtès, ouvr. cité, p. 137).

42. Sur ce point je rejoins Bruno Bettelheim (ouvr. cité, p. 396) : «[...] la nature brutale des deux sœurs; elles sont prêtes à tout pour frustrer [Aschentputtel] et atteindre leur but.» D'ailleurs, concernant la cruauté des sœurs d'Aschenputtel versus celles de Cendrillon, remarquons, à la suite de la discussion de la note 27 , que le thème de la mutilation est présent dans le "Peau d'Âne» de Perrault («L'autre en coupe un petit morceau / [...] Pour faire que son doigt cadre bien à l'anneau»), attestant que les sœurs de Cendrillon, au-delà des styles respectifs des auteurs Perrault et Grimm, ne sont pas portées à de telles extrémités.

43. Dans le sens de la remarque de Bruno Bettelheim. 
le conte «Cendrillon» : le prince ne sort pas de son palais, ni pour poursuivre la jeune femme ni pour la retrouver (il envoie un émissaire pour cela), contrairement à celui d'Aschenputtel (l. 20); l'héroïne une fois reconnue est «encore plus magnifique[ment parée] " pour les retrouvailles, contrairement à celles plus spirituelles d'Aschenputtel (1. 23). Insistons sur ce point, si la recherche de la beauté de l'âme peut être une éthique présente dans les contes de Grimm, les contes-de-Perrault proposent une dialectique de l'être et du paraître plus socialement pragmatique, où le paraître n'est pas stigmatisé comme un "état de mensonge ${ }^{44}$ ", que cela soit avec "Cendrillon», mais aussi avec "Le Chat botté» ou «Riquet à la houppe».

Le récit "Aschenputtel» des Grimm est très différent du conte «Cendrillon» de Perrault; l'absence d'interdit dans le premier y est d'une grande cohérence narrative, tout comme sa présence dans le second. Ainsi, en conclusion de cette première étude, l'interdit de minuit s'avère receler, plutôt qu'une leçon de morale, une leçon de vie initiatique. Et nous avons montré que la marraine l'a posé délibérément dans un but salutaire, voire pédagogique.

\section{Le cas des frères-cygnes}

Dans le conte «Les six cygnes» des Grimm ${ }^{45}$, six frères ont été transformés en cygnes par une sorcière. Un jour, leur sœur unique et aimante les retrouve et leur demande s'il existe un moyen de les rendre à nouveau humains. Voici la réponse :

Hélas non, dirent-ils, les conditions sont trop difficiles. Pendant six ans, tu n'auras pas le droit de parler ni de rire, et tu devras coudre pour nous, pendant ce temps, des chemises d'asters. Si un seul mot venait à sortir de ta bouche, tout ton travail aura été vain.

Il s'agit donc d'une double condition : un travail de couture et une règle de mutisme.

\section{Le silence est d'or}

La sanction est en partie explicite. On comprend bien qu'en cas de rupture du mutisme, en dépit du travail effectué, il ne sera plus jamais pos-

44. J. Courtès, ouvr. cité, p. 133 .

45. J'utilise la traduction de Natacha Rimasson-Fertin (J. et W. Grimm, Contes pour les enfants et la maison, 2009, p. 275-279). 
sible de délivrer les frères de leur ensorcellement (ou bien, au mieux, elle devra reprendre le travail au début, mais l'ambiguïté indique que cela ne fait pas de grande différence étant donné la difficulté de la chose); mais on ne sait pas au juste, dans le cas d'un mutisme respecté mais d'un nombre insuffisant de chemises tissées à l'échéance des six ans, ce qu'il adviendra. On peut supposer qu'alors les chemises réalisées seront néanmoins performantes et que seuls ceux qui en bénéficieront seront délivrés (comme la fin du conte le prouvera avec une aile qui subsiste à cause d'une manche manquante). Ainsi conçues, les conditions sont optimales : ce qui garantit que la sœur s'efforcera de respecter l'échéance pour l'ensemble des chemises requises est son affection égale pour tous ses frères; et ce qui garantit qu'elle ne rompra pas même une fois son vœu de mutisme est son propre intérêt à ne pas œuvrer pour rien.

Mais ici aussi, à l'instar de ce que nous avons examiné dans le cas de "Cendrillon», aucun motif n'est donné.

Concernant la première instruction - le travail de couture-, on comprend assez bien l'idée de préparer avec les tuniques une nouvelle peau capable de métamorphoser les frères magiquement et de leur redonner leur peau originelle. D'ailleurs il ne s'agit que d'inverser l'ensorcellement initial réalisé avec des chemises de soie blanche, une sorte d'antidote symbolique.

Mais pourquoi cet interdit de parler et de rire, pourquoi cette obligation de mutisme? Ici aussi, comme nous l'avions fait dans le cas de "Cendrillon", écartons les explications postulant par principe des invraisemblances propres au genre littéraire. S'agirait-il alors de rendre l'épreuve plus difficile? La tâche essentielle est déjà suffisamment pénible en soi et demande une longue abnégation (six ans!). Au fond, ce qui est demandé à la sœur, c'est un incroyable geste d'amour pour inverser le geste de haine initial de la marâtre. Et c'est bien pourquoi les frères ne présentent ces conditions, avec à la fois délicatesse et astuce, que comme des règles magiques factuelles, et non comme une demande qu'ils adresseraient à leur sœur. Ils n'énoncent les conditions qu'avec réticence et qu'après qu'elle le leur ait demandé alors même qu'ils les connaissaient déjà ; mais en les présentant d'emblée comme impossibles à réaliser, par une sorte de déni rhétorique, ils mettent leur sœur au défi ${ }^{46}$.

46. En fait, un examen attentif des éditions successives des Contes pour les enfants et la maison des Grimm montre que le passage qui nous occupe est le résultat d'une évolution génétique, majeure entre I8I2 et I8I9. Après que les frères ont expliqué à leur sœur leur métamorphose vespérale, nous trouvons (je traduis) : en I812, "Si tu veux nous délivrer, tu dois [...]»; en I819, "Mais je ne peux pas vous délivrer? dit la jeune fille. Hélas non, répondirent-ils, tu ne peux pas, car c'est trop difficile : [...]»; en I837 (et suiv.), «Leur sœurette se mit à pleurer et dit : vous ne pouvez donc pas 
Maria Tatar, dans un chapitre sur les contes ayant trait au tissage, considère que l'héroïne "finds deliverance by withdrawing from the world and retreating into the sphere of silent domestic activities ${ }^{47}$ ". Sans conserver l'argument du personnage féminin renvoyé inéluctablement à la tâche domestique, François Flahault poursuit la première idée et propose de comprendre le silence de manière métaphorique : "Le silence de l'héroïne est analogue à ce long tissage médiateur : il la rapproche de l'inhumain, chose nécessaire pour permettre à l'inhumain de redevenir humain ${ }^{48}$." Quant à nous, commençons par envisager la situation avec pragmatisme.

Imaginons ainsi, en guise de raisonnement narratif, ce qui se passerait si seule la condition du travail de couture était imposée. Bien sûr, la sœur, si dévouée, se mettrait au travail avec détermination, isolée dans la forêt. D'ailleurs, jusque-là, «elle n'a[ur]ait personne à qui parler et elle n'a[ur] ait pas envie de rire; elle rest[er] ait assise là et ne lev[er]ait pas les yeux de son travail». Puis lorsque surviendrait le groupe de chasseurs, intrigués par cette jeune fille seule, cousant des asters, l'assaillant de questions, il lui faudrait bien parler. Elle expliquerait sa tâche étrange face à des visages incrédules, sans parvenir à les convaincre : après tout, d'où tient-elle que les tuniques d'asters auront un pouvoir magique? Ses frères le lui ont-ils affirmé? Mais d'où eux-mêmes le tiennent-ils? Elle devra bien convenir qu'elle n'en sait rien... Ils l'emmèneraient de force, sa beauté attirerait l'attention du prince... Que se passerait-il alors? Au pire on la prendrait pour une sorcière (ce qui se produira d'ailleurs immanquablement avec le scénario réel ${ }^{49}$ mutique), au mieux on s'efforcerait dans des discussions interminables de la dissuader de poursuivre un tel labeur apparemment si vain; elle courrait le risque qu'on lui instille le doute et le découragement; peut-être même le prince serait-il capable de la faire rire. Bref, elle serait au pire interrompue et au mieux seulement ralentie dans son travail de couture, elle piétinerait dans sa mission de délivrance, or le temps lui est comptéso.

être délivrés? Hélas non, répondirent-ils, les conditions sont trop difficiles : [...]». Ainsi, c'est à partir de la deuxième édition que les frères n'énoncent pas spontanément les conditions de leur délivrance mais en réponse à une question spécifique de leur sœur, et que la formulation, d'abord neutre, se transforme en ce déni rhétorique. Puis, à partir de 1837 , la forme se stabilise : la question de leur sœur est plus apitoyante et le terme de "conditions» est introduit.

47. M. Tatar, The Hard Facts of the Grimms' Fairy Tales, 2003, p. II5.

48. Fr. Flahault, La Pensée des contes, 200I, p. I36.

49. Par réel, il faut entendre ici «fictif» opposé à «imaginaire», c'est-à-dire le récit des Grimm, réel dans sa réalité fictive, par rapport à mon scénario imaginaire ci-dessus.

50. D’ailleurs, notons que même lorsqu'elle ne dit mot, dans le scénario réel, elle achève à peine le travail de couture au tout dernier moment de l'échéance. 
Il apparaît ainsi que l'interdit de communiquer qui est proposé à la sœur l'oblige à consacrer toute son attention à sa tâche, sans dévier un seul instant, sans se laisser divertir : "Elle restait assise là et ne levait pas les yeux de son travail»; "elle restait assise là, à coudre ses chemises, et ne prêtait attention à rien d'autre». Après tout, le vœu de silence monastique n'a-t-il pas aussi pour but de purifier l'esprit de toute distraction? Ainsi, en cessant de parler et de rire, donc de communiquer avec les humains, la sœur est en effet amenée à se rapprocher de l'inhumanité de ses frères, comme l'a remarqué Flahault.

Cependant, à l'inverse du scénario imaginaire envisagé plus haut où il ne serait pas interdit à la sœur de communiquer, mais aboutissant à l'échec de sa mission salvatrice, force est de constater que son mutisme lui attire des ennuis et la met finalement en danger. On imagine sa souffrance quand, mise en accusation inique, elle décide de rester coite. Il est raisonnable de penser que si, communicante, la sœur prend le risque d'échouer à sauver ses frères, mutique, elle améliore ses chances de tenir les délais et donc de les sauver mais augmente d'autant son risque personnel.

Son silence est donc une condition de réussite de l'épreuve, qui est de coudre, aussi risqué cela soit-il pour elle. Cet interdit est donc ici aussi salutaire.

\section{Deux conditions intriquées}

Cyrille François (20I2) a établi la parenté, et même la filiation, entre notre conte (B) et trois autres récits allemand et danois : Die sieben Schwäne (anonyme), De elleve Svaner de Mathias Winther et De vilde Svaner de Hans Christian Andersen. Il est révélateur de comparer les conditions qui sont posées à la sœur pour désensorceler ses frères.

Concernant la confection des chemises, il s'agit bien entendu d'en coudre autant que de frères. La difficulté réside, dans les contes $\mathrm{A}$ et $\mathrm{B}$, dans la durée longue mais limitée de l'épreuve, montrant bien qu'une chemise est censée être tissée en un an (ce qui atteste de la difficulté de l'épreuve); tandis que pour les contes $\mathrm{C}$ et $\mathrm{D}$, aucun délai n'est fixé mais le tissage doit se faire à partir de plantes piquantes et urticantes, dont le moyen d'obtention est lui-même périlleux dans le cas du récit $\mathrm{D}$. Sans doute que cette pénibilité supplémentaire compense une durée plus réduite, car prévoir onze et douze ans serait narrativement problématique. Quoi qu'il en soit, 
il s'agit bien d'établir l'ampleur du calvaire demandé et donc le dévouement admirable, voire sublime et sacrificiel ${ }^{\text {sI }}$, de la sœur.

Tableau $\mathbf{n}^{\circ} 2$

\begin{tabular}{|c|c|c|c|c|c|}
\hline Récit & & A & B & $\mathrm{C}$ & $\mathrm{D}$ \\
\hline \multirow{5}{*}{ Description } & Titre original & $\begin{array}{l}\text { Die sieben } \\
\text { Schwäne }\end{array}$ & $\begin{array}{l}\text { Die sechs } \\
\text { Schwäne }\end{array}$ & $\begin{array}{l}\text { De elleve } \\
\text { Svaner }\end{array}$ & De vilde Svaner \\
\hline & Titre français & $\begin{array}{l}\text { «Les sept } \\
\text { cygnes» }\end{array}$ & «Les six cygnes» & $\begin{array}{l}\text { «Les onze } \\
\text { cygnes» }\end{array}$ & $\begin{array}{l}\text { «Les cygnes } \\
\text { sauvages» }\end{array}$ \\
\hline & $\mathrm{I}^{\mathrm{re}}$ édition & I8OI & I8I2 & 1823 & I 838 \\
\hline & Langue & allemand & allemand & danois & danois \\
\hline & Auteur & Anonyme & J. et W. Grimm & M. Winther & H. C. Andersen \\
\hline \multirow{2}{*}{ Analyse } & Destinateur & & frères & $\begin{array}{l}\text { frère aîné en } \\
\text { rêve }\end{array}$ & $\begin{array}{c}\text { fée Morgane en } \\
\text { rêve }\end{array}$ \\
\hline & $\mathrm{Nb}$ de frères & 7 & 6 & II & $\mathrm{I} 2$ \\
\hline \multirow{3}{*}{$\begin{array}{l}\text { Condition } \\
n^{\circ}{ }^{\circ}\end{array}$} & Couture & & en fleurs d'aster & en chardons & $\begin{array}{l}\text { en orties de } \\
\text { cimetières }\end{array}$ \\
\hline & Échéance & 7 ans & 6 ans & I & I \\
\hline & Transgression & & $\begin{array}{l}\text { une manche > } \\
\text { une aile subsiste }\end{array}$ & & $\begin{array}{c}\text { une manche > } \\
\text { une aile subsiste }\end{array}$ \\
\hline \multirow{3}{*}{$\begin{array}{l}\text { Condition } \\
\mathrm{n}^{\circ} 2\end{array}$} & Silence & ni pleurer & ni rire & I & I \\
\hline & Sanction & & vain & I & mort des frères \\
\hline & Transgression & $\begin{array}{c}\text { une larme > } \\
\text { perte d'un œil }\end{array}$ & I & I & tentations \\
\hline
\end{tabular}

Concernant la condition complémentaire — l'interdit de communiquer - , elle subit aussi quelques variations qui sont intrinsèquement liées à la condition première. Si dans le conte $\mathrm{B}$, comme nous l'avons vu, il est imposé de ni parler ni rire, dans le conte $\mathrm{A}$ il s'agit de ne pas parler ni pleurer, tandis que les récits $\mathrm{C}$ et $\mathrm{D}$ s'en tiennent au seul silence. Il semblerait que si le conte B craint que la sœur ne retrouve, grâce au prince,

5I. «[Elle] résolut [...] de délivrer ses frères, quand bien même elle devrait le payer de sa propre vie» et de la confiscation de ses propres enfants. À comparer avec l'attitude moins sacrificielle de la sœur dans le récit apparenté "Les sept corbeaux» des Grimm, limitée à son petit doigt, et motivée par un sentiment de culpabilité envers ses frères. 
une joie de vivre qui la distrairait de son labeur, le conte A envisage plutôt qu'elle puisse désespérer elle-même et ainsi s'interrompre; tandis que les récits $\mathrm{C}$ et $\mathrm{D}$, dont la pénibilité de la condition première a été accrue, ne se prêtent pas au (sou) rire ${ }^{52}$ et ne peuvent réprimer les pleurs (de sorte que la nouvelle $\mathrm{D}$ présente une jeune fille quasiment sainte). Ainsi, la formulation de l'interdit salutaire s'adapte à ce qui pourrait représenter un obstacle psychologique à la réalisation de la mission salvatrice, en fonction de la difficulté de la condition première.

La nouvelle $\mathrm{D}$ amplifie même les enjeux de l'interdit salutaire, puisque la sanction, en cas de rupture du mutisme, ne se limite pas au statu quo mortifère comme avec le conte $\mathrm{B}$, mais menace littéralement de mort ses frères : «Le premier mot sorti de ta bouche atteindrait le cœur de tes frères comme un poignard mortel. Ainsi, leur vie dépend de ta langue.» En utilisant une métaphore visuelle frappante plutôt que le simple verbe «tuer», la possibilité que la sœur rompe son mutisme est encore minimisée, comme l'atteste le fait qu'elle se remémore régulièrement le danger : "[plutôt que de répondre à une question qui lui est adressée, elle] secoua la tête, car la vie de ses frères dépendait de son silence»; "Quoique sa bouche fût muette, puisqu'une seule parole leur eût coûté la vie». D'ailleurs ce récit souligne plus encore les tentations de rupture du mutisme : "Tous les jours elle aimait [le roi] de plus en plus : aussi elle aurait pu se confier à lui et lui raconter ses souffrances, mais il fallait qu' elle restât muette pour mener son œuvre à bonne fin.»

\section{Le fonctionnement de l'interdit}

L'examen attentif et rigoureux des interdits (minuit et mutisme) dans ces deux récits distincts, "Cendrillon» et "Les six cygnes», nous a permis de montrer que l'interprétation la plus simple de leur fonction consiste, dans les deux cas, en une aide salutaire à la réussite de l'objectif principal des héroïnes (séduire et coudre).

Remarquons que nos interdits s'écartent du schéma proppien. Dans les deux cas, ils sont posés aux hérö̈nes par des personnages qui leur sont foncièrement bienveillants (d'une part la marraine, d'autre part les frères, qui sont même directement intéressés à la réussite de leur sœur); dans les deux cas, les interdits ne sont pas du tout transgressés.

52. Récit D : «Le roi $[\ldots]$ fit jouer de la musique [...]. Cependant aucun sourire ne parut sur ses lèvres ou dans ses yeux; la douleur seule s'y montrait." 
Mais alors, en admettant notre thèse d'interdits posés délibérément par des adjuvants ne visant qu'à procurer une méthode pour la réussite des héroïnes, se présentent deux questions : I) pourquoi ces adjuvants ne pouvaient-il pas simplement proposer leurs idées sur la meilleure manière de réussir l'épreuve sous forme de simples conseils? 2) pourquoi ces personnages supposés si favorables ne pouvaient-ils pas expliquer clairement aux héroïnes les raisons de tels interdits?

\section{L'interdit et sa sanction}

Nous avons déjà noté l'ambiguïté des formulations, dans nos récits, quant à leur statut "d'interdit». Dans "Cendrillon», il s'agit d'une «recommandation", et dans "Les six cygnes», il s'agit d'une "condition ${ }^{53}$ ». Aussi, pourquoi ne pas avoir formulé clairement des conseils ${ }^{54}$ ? Par exemple, cela aurait pu donner :

- pour "Cendrillon» : ne reste pas trop longtemps au bal, sinon tu lasseras le prince; pars au moment où tu rencontreras le plus de succès, avant que les danses ne s'achèvent, sans doute vers minuit, sinon tu ne pourras pas emporter durablement le cour de ton amant;

- pour "Les six cygnes" : la tâche est si difficile qu'il te faudra t'y consacrer entièrement et te concentrer sans la moindre distraction, le mieux serait que tu t'abstiennes de parler avec quiconque, sinon, dissuadée de poursuivre ou seulement ralentie, tu échoueras.

Bien sûr, les héroïnes protesteraient de bonne foi de leur volonté de bien faire et de leur discernement à mettre en ouvre la plus sage manière d'atteindre leurs objectifs. Elles assureraient qu'elles comprennent parfaitement le bon sens et le caractère judicieux de ces conseils, et qu'elles feront de leur mieux. Mais imaginons (par un récit alternatif) ce qui se passerait alors :

- pour «Cendrillon» :

Lorsqu'elle entendit sonner le premier coup de minuit, elle poussa un soupir, résignée à partir, mais au moment où elle se détournait vers l'entrée du palais, elle repéra cette si gracieuse princesse, dont on disait qu'elle avait eu les plus grands maitres à danser, qui s'approchait du prince. Alors que les violons commencèrent à jouer la première

53. Mais la notion d'interdit est explicitement évoquée deux fois : lors de l'énonciation initiale de la condition ( du darfst nicht» que Natacha Rimasson-Fertin traduit par «tu n'auras pas le droit»), puis en fin du récit ("nicht ... durfte» que Natacha Rimasson-Fertin traduit par «il était interdit»).

54. Comme, dans les contes-de-Perrault, on en trouve dans «Peau d'Âne» («Pourvu qu’à mes conseils vous vous laissiez conduire»), dans «Le Chat Botté» («Si vous voulez suivre mon conseil, votre fortune est faite») et «Le Petit Poucet» («Ils crurent son conseil»). 
mesure d'un menuet, Cendrillon reprit soudainement la main du prince, se disant qu'elle pourrait bien s'en aller à la fin de cette danse. Mais à l'issue de celle-ci, à nouveau elle trouva une raison de s'attarder. Bientôt il fit jour...

- pour «Les six cygnes»:

Lorsque survint un groupe de chasseurs, la sœur, se remémorant le conseil reçu, resta coite. Ils pouvaient bien la croire muette, seul lui importait de sauver ses frères. Puis on l'emmena auprès du roi qui succomba à son charme, et elle devint reine. Elle s'efforçait de ne pas lever ses yeux de son ouvrage mais il faut dire qu'il était beau, et surtout qu'il était bon et doux avec elle. Elle résistait à l'envie de se confier à lui, mais elle le voyait tant souffrir des médisances à son sujet, qu'elle se dit qu'elle pourrait bien lui parler juste quelques instants pour s'expliquer, sans se retarder trop. Il fut très surpris quand elle ouvrit la bouche mais il écouta ses paroles et comprit ses raisons. Seulement désormais, dans le seul but d'abréger les souffrances de sa reine, le roi venait l'entretenir sans cesse de ses bonnes idées pour délivrer ses frères par d'autres moyens, et elle devait bien se résoudre à lui répondre. Lorsqu'il ne fut plus possible de mettre fin à la cabale à son encontre et que le procès commença, le roi la conjura de parler, ce qu'elle fit, de sorte qu'arriva vite le dernier jour des six ans, et qu'elle n'avait qu'à peine avancé dans sa tâche.

En effet, n'est-il pas évident que la formulation d'un conseil, comportant même une description des conséquences naturelles"s qui se produiraient s'il n'était pas suivi, offre moins de garanties de respect scrupuleux qu'un interdit, c'est-à-dire une prescription assortie d'une sanction en cas d'infraction ${ }^{56}$ ? Surtout si le sujet concerné n'a pas encore acquis une maturité suffisante pour intégrer de lui-même la pertinence du comportement demandé.

Soit, mais pourrait-on alors penser que, de la part de protecteurs, des menaces de sanctions suffiraient pour inciter les deux hérö̈nes à respecter ces «conseils» et qu'il ne serait pas nécessaire d'avoir vraiment l'intention de les appliquer? Le conte «Les six cygnes», de par sa construction narrative, ne permet pas de le vérifier. En tout cas il semble que l'héroïne (et le lecteur aussi sans doute) soit suffisamment persuadée que ce ne sont pas des vaines menaces et elle ne les prend pas à la légère : elle ne courra pas le risque de rompre, même une fois, son mutisme. Mais le conte «Cendrillon» offre l'occasion à l'héroïne de vérifier par deux fois la réalité de la sanction :

55. L'alternative crédible à la sanction d'un interdit, afin d'être respecté, étant bien sûr de convaincre de la certitude de conséquences surnaturelles, comme le font les marraines de Cinderella (voir note 5). D'ailleurs, dans Cinderella de Disney, la marraine n'énonce même pas de règle à suivre à sa destinataire, il lui suffit d'évoquer la conséquence qu'il y aurait à rester après minuit.

56. La sanction délibérée de l'énonciateur, et non pas la conséquence naturelle indépendante de lui, est en effet essentielle à la définition d'un interdit. Hans Kelsen établissait déjà, dans sa Théorie pure du droit, que la sanction caractérise la norme juridique (1988, p. 70). 
"Cendrillon arriva chez elle [...], sans carrosse, sans laquais, et avec ses méchants habits, rien ne lui étant resté de toute sa magnificence.» On comprend bien que, par souci de crédibilité, la marraine ne peut pas se permettre de ne pas exécuter l'avertissement annoncé.

Dira-t-on que les émetteurs des interdits, pourtant bienveillants envers les héroïnes, leur feraient prendre des risques bien importants si on admet qu'ils mettent délibérément et réellement en place ces sanctions? En effet, en cas de transgression, Cendrillon, si elle outrepassait minuit, devrait non seulement affronter un ridicule particulièrement embarrassant, sans doute un rejet du prince, et, ultérieurement, des brimades familiales. Quant à la sœur, en cas de rupture du mutisme, elle condamnerait ses frères à leur destin animal (dans le récit des Grimm), voire à la mort (dans le récit d'Andersen). Comment comprendre que des personnages positifs puissent faire prendre de tels risques à leurs protégés?

Il s'agit là du résultat d'un calcul somme toute rationnel ${ }^{57}$. Dans le cas de la sœur des frères-cygnes, sans interdit salutaire, au mieux parviendraitelle à tisser quelques chemises et donc à sauver quelques-uns de ses frères, soit un gain nul à moyen; inversement, soumise à l'interdit de communiquer, au pire elle le transgresse et se retrouve dans la situation initiale (dans le récit des Grimm), soit une perte faible ${ }^{58}$ (en intégrant la culpabilité due à son échec), au mieux elle respecte le mutisme et maximise ainsi ses chances de les sauver tous ${ }^{59}$ : gain maximal. Il faut considérer aussi que la sœur, avec candeur, compte sincèrement faire de son mieux sans anticiper ou même imaginer des difficultés qui pourraient survenir, contrairement aux personnages plus expérimentés que sont ses frères (ou la fée dans le récit d'Andersen).

Dans le cas de Cendrillon, sans interdit salutaire, elle n'optimise pas ses chances de séduire le prince et de sortir de sa condition misérable, mais passe certainement un moment mémorable à ce bal, soit un gain faible; inversement, soumise à l'interdit de minuit, au pire elle le transgresse et se

57. Nous ne prétendons pas recourir, dans l'analyse qui suit, au formalisme de la théorie des jeux, mais simplement faire œuvre de logique.

58. Dans le cas du récit d'Andersen, la transgression entraînerait une perte forte, la mort, mais pas maximale, à considérer que leur situation animale est déjà assez mortifère en soi; c'est pourquoi, dans ce récit, la formulation de l'interdit exprime plus clairement qu'il ressort pour l'héroïne d'un choix de se lancer ou non dans une entreprise aussi risquée : «Rappelle-toi bien que, depuis le moment où tu auras commencé ce travail jusqu'à celui où il sera terminé [...] il te faudra garder un silence absolu. [...] leur vie dépend de ta langue.» (je souligne)

59. Bien que prenant alors le risque d'être elle-même exécutée comme sorcière : mais n'est-elle pas justement sublime d'abnégation? 
retrouve dans la situation initiale, avec quelques embarras au passage, soit une perte faible; au mieux elle fait preuve de bonne grâce avec virtuosité, et emporte le gain maximum : in fine elle peut devenir reine. Il est même légitime de penser que si Cendrillon n'a espéré, en allant au bal, que de vivre un moment extraordinaire, et peut-être dans le meilleur des cas se trouver un gentilhomme de mari, sa marraine, elle, a anticipé, connaissant les qualités de sa filleule, la possibilité du gain maximal, séduire le prince, lors de cette occasion unique, et donc pesé les risques conformément.

Il est donc tout à fait rationnel pour des personnages bienveillants de prendre de tels risques envers leurs protégés en choisissant une stratégie d'interdits salutaires.

\section{Linterdit et son motif}

Pourrait-on concevoir que les destinateurs, tout en posant leurs interdits, prennent soin de les motiver, de les expliquer? Mais du même coup serait révélé clairement que ces interdits ne correspondent à aucune contrainte techniquement magique, et qu'ils sont posés délibérément dans l'intention d'obliger à une bonne pratique. On imagine aisément les réactions de protestation des destinataires : «Ne vous inquiétez pas! Nous avons compris la sagesse de vos conseils, nul besoin d'une menace...» Et même si elles en acceptaient le principe, la perspective de transgresser les interdits leur semblerait moins grave, possiblement négociable pour une seconde chance.

Il fallait donc bien, du point de vue des personnages adjuvants, surtout ne pas discuter des motifs de leurs interdits, que cela reste à leur insu, tout au moins initialement. Aussi s'arrangent-ils pour ne pas avoir à fournir d'explication et pour limiter la possibilité qu'il leur en soit demandé.

Ainsi, dans le cas de "Cendrillon", la marraine énonce-t-elle la règle in extremis, une fois la jeune fille parée et montée en carrosse, et évidemment trop impatiente de partir ("ne se sentant pas de joie») pour entamer une discussion ${ }^{60}$. D'ailleurs, sa marraine ne lui avait-elle pas demandé d'emblée d'être "bonne fille», comme condition à sa coopération («seras-tu bonne fille? dit sa Marraine, je t'y ferai aller»), c'est-à-dire ici ${ }^{61}$ de lui vouer une confiance un peu aveugle (non pas «sois gentille» mais «faismoi confiance, crois-moi sur parole, ne [me] pose pas de question»). Confiance dont Cendrillon fait preuve immédiatement : "Apporte-moi

6o. Ou, dans le cas de Cinderella de Disney, trop reconnaissante déjà de pouvoir rien qu'un peu aller au bal pour oser sembler contester.

6I. Dans le sens "qui ne songe à aucune malice" (Dictionnaire universel de Furetière, 1690, Bon номме) qui implique une confiance à la limite de la crédulité. 
une citrouille. Cendrillon alla aussitôt cueillir la plus belle [...], et la porta à sa Marraine, ne pouvant deviner comment cette citrouille la pourrait faire aller au Bal»; ce qui signifie précisément que Cendrillon se le demande mais ne le demande pas.

Dans le cas des frères-cygnes, le dispositif par lequel l'héroïne apprend les conditions de la délivrance de ses frères fait écran à toute interrogation éventuelle. Soit, dans le conte B, ce sont les frères qui s'expriment collectivement ${ }^{62}$, et, comme nous l'avons déjà vu, mettent leur sœur au défi, concentrant son attention sur sa détermination plutôt que sur sa rationalité, et de toutes les manières s'éclipsent aussitôt achevé leur discours; soit, dans les récits $\mathrm{C}$ et $\mathrm{D}$, les conditions sont communiquées durant un rêve, de telle sorte que la sœur n'est jamais en contact réel et direct avec le supposé émetteur initial.

\section{Linterdit pédagogique}

L'interdit, que l'on peut définir maintenant comme une prescription sanctionnée mais non motivée (au contraire du conseil), correspond bien à la forme requise dans les situations de nos récits. Pourtant, les deux cas étudiés diffèrent sur un point important.

L'enjeu essentiel du conte "Les six cygnes» des Grimm étant que la sœur fasse preuve d'une affection rédemptrice envers ses frères, l'objectif unique est qu'elle réussisse l'épreuve. Il ne s'agit pas de lui inculquer la vertu de la concentration en toutes choses! De fait, la sœur dévouée des frères-cygnes ne semble pas réaliser que la condition de mutisme qui lui est imposé n'est pas une fin en soi mais un moyen de sa réussite ${ }^{63}$.

Tandis que dans le conte "Cendrillon» de Perrault, l'enjeu est celui d'une initiation, d'un apprentissage ${ }^{64}$. Mais le personnage Cendrillon doit le

62. L'usage du "dirent-ils» est bien entendu métaphorique : qu'ils s'expriment vocalement à l'unisson ou complètent le discours les uns les autres, en tout cas ils donnent le sentiment à leur sœur d'être tous d'accord avec l'énoncé, rendant cela indubitable, de sorte qu'elle ne les questionne pas sur l'origine de ces informations.

63. Néanmoins, dans le récit d'Andersen, novella trois fois plus longue que le conte des Grimm (I85I et 5913 mots respectivement), l'héroïne semble évoluer au cours de son long labeur : elle évoque la nécessité de rester silencieuse à trois reprises, les deux premières fois en le justifiant par la conséquence pour ses frères ("car la vie de ses frères dépendait de son silence», "puisqu'une seule parole leur eût coûté la vie»), puis la dernière fois car «il fallait qu'elle restât muette pour mener son œuvre à bonne fin".

64. D'ailleurs, l'évolution du personnage se remarque aussi par une analyse fine de la représentation de sa parole entre discours directs et indirects, comme le montre avec brio Jean-Michel Adam (ouvr. cité, p. 287) : elle "progresse [d'un discours émotionnel] vers la maitrise rhétorique de la parole». 
découvrir par elle-même. De fait, sa marraine lui laisse matière à réfléchir, lors de sa préparation pour se rendre au bal. Outre le mystère entretenu à dessein sur l'interdit de minuit, la nature des pantoufles est au moins aussi mystérieuse. D'abord, sa marraine les lui remet telles quelles, objets déjà en soi extraordinaires, contrairement à tous les autres éléments de sa parure qui résultent d'une transformation magique; ensuite, sa marraine ne les mentionne pas lorsqu'elle «l'averti[t] que si elle demeurait au Bal un moment davantage, son carrosse redeviendrait citrouille, ses chevaux des souris, ses laquais des lézards, et que ses vieux habits reprendraient leur première forme»; enfin, Cendrillon peut constater d'elle-même, passé minuit, qu'en effet tout reprend sa première forme, hormis les pantoufles qui subsistent. Certes, même un lecteur attentif pourrait, à sa première lecture, ne pas remarquer ces anomalies, en revanche la protagoniste, qui visualise non seulement parfaitement la situation mais a participé à la collecte des objets de transformation, ne peut pas les avoir manquées. Réfléchie comme elle est, plutôt que d'interroger sa marraine à ce sujet, même ultérieurement, gageons qu'elle réalisera que cela est ainsi à dessein ${ }^{65}$, et en tirera les conséquences : elle abandonne délibérément ses pantoufles, puisqu'elle a compris qu'il s'agit du seul signe qu'elle peut laisser ${ }^{66}$.

Cendrillon a intégré d'autant plus pleinement l'enseignement de sa marraine qu'elle l'a expérimenté (le premier soir) avant que d'en comprendre la leçon par elle-même. C'est pourquoi le deuxième soir, Cendrillon «s'enfuit aussi légèrement qu'aurait fait une biche» et non dans la frustration ou l'angoisse : douée de bonne grâce, elle connaît son effet. De ce point de vue, l'interdit auquel elle est soumise est non seulement salutaire, mais porte une valeur pédagogique qui lui permet de résoudre le paradoxe d'une bonne grâce apprise naturellement.

\section{Conclusion}

Létude de deux interdits dans deux contes merveilleux distincts nous permet de réfuter les seules fonctions dramatique et probatoire que la critique leur accorde généralement. Nous avons pu montrer plutôt que ces interdits-là partagent une intention similaire, celle de viser le salut des destinataires.

65. Comme l'avait déjà remarqué Marc Escola (Marc Escola commente Contes de Charles Perrault, 2005, p. 140).

66. À l'instar de sa voisine antérieure des contes-de-Perrault, Peau d'Âne, qui enfouit sa bague dans une galette. 
Ils se distinguent cependant sur la valeur pédagogique que comporte l'interdit dans "Cendrillon", contrairement à celui des "Six cygnes». Nous validons ainsi la fécondité de l'analyse du fonctionnement des interdits par le triplet \{règle - motif - sanction\}.

Il en résulte qu'avec une virtuosité si à propos, Perrault élabore une mise en abyme d'une grande cohérence discursive. En effet, ce que la marraine tente d'enseigner à l'héroïne, soit un certain art de l'ambiguïté, elle le pratique à son égard, et le texte nous narre cela de la même manière : Cendrillon expérimente une pratique de voilement/dévoilement pour séduire le prince (entre son nom, caché, son apparence, éblouissante, ses pieds, apparents à travers le verre lorsque sa robe se soulève, ses disparitions, inopinées); la marraine transmet son message éducatif à Cendrillon sous la forme d'un interdit lui permettant de s'en approprier par l'expérience le sens caché; le texte représente au lecteur cet enseignement sous la forme narrative d'un conte plein d'ambiguïtés (du motif de l'interdit à l'abandon de la pantoufle), plutôt qu'une forme argumentative, sans didactisme.

Il serait intéressant d'étudier si ce type d'analyse permet de réinterpréter les récits merveilleux de Sodome et Gomorrhe et d'Orphée et Eurydice: les interdictions de se retourner possèdent-elles également une fonction salutaire? Plus généralement, il apparaît que l'analyse fine de l'interdit peut permettre de distinguer plusieurs types d'interdits, et que le corpus des contes merveilleux se prête idéalement à ce programme de recherche.

\section{Bibliographie}

\section{Textes}

Andersen Hans Christian, Contes, trad. D. Soldi [1856], Paris, Librairie Hachette et $\mathrm{C}^{\mathrm{ie}}$, I876, p. 302-329.

Grimm Jacob \& Wilhelm, Kinder- und Hausmärchen. Gesammelt durch die Briider Grimm, H. Rölleke (éd.), 2 vol., Göttingen, Vandenhoeck \& Ruprecht, 1986

Grimm Jacob et Wilhelm, Contes pour les enfants et la maison, édités et traduits par N. Rimasson-Fertin, Paris, José Corti, 2009, vol. I.

Marzolph Ulrich (éd.), Feen-Mährchen: zur Unterhaltung für Freunde und Freundinnen der Feenwelt. Volkskundliche Quellen, Hildesheim, Olms, 2000.

Perrault Charles, Contes, établis par G. Rouger [1697], Paris, Garnier, I967. 
Winther Mathias, Danske Folkeeventyr, Copenhague, Den Wahlske Bohandlings Forlag, I823.

\section{Études}

Adam Jean-Michel, «Le dialogue intertextuel du Trésor des Contes d'Henri Pourrat avec les Grimm et Perrault», Féeries, nº 9, "Le dialogue intertextuel des contes de Grimm», 20I2, p. I6I-I96.

Adam Jean-Michel et Heidmann Ute, Textualité et intertextualité des contes, Paris, Classiques Garnier, 2010.

Bettelheim Bruno, Psychanalyse des contes de fées [1976], Paris, Robert Laffont, coll. «Pluriel», 2007.

Bricout Bernadette, La clé des contes, Paris, Seuil, 2005.

Calvino Italo, Contes populaires italiens, t. 4 : Les Îles, Paris, Denoël, I984. CourTès Joseph, Introduction à la sémiotique narrative et discursive [1976], Paris, Hachette, I980.

Du Bosc Jacques, L'Honnête femme [1632], Paris, Compagnie des Libraires du Palais, I665.

Escola Marc, Marc Escola commente Contes de Charles Perrault, Paris, Gallimard, coll. "Foliothèque», 2005.

FARET Nicolas, L'honnête homme ou l'art de plaire à la Cour, Paris, Toussaint du Bray, I6zo.

Flahault François, La Pensée des contes, Paris, Anthropos, coll. «Economica», 200I.

Françors Cyrille, "Échanges et dialogues intertextuels : l'exemple des contes de Perrault et des frères Grimm ", dans $\mathrm{M}$. Bragança et $\mathrm{S}$. Wilson (éds), É/change : transitions et transactions dans la littérature française I Ex/change: Transitions et Transactions in French Literature, Oxford, Peter Lang, 20II, p. 43-57.

Françors Cyrille, "C'est la plume qui fait le conte : Die sechs Schwäne des frères Grimm et De vilde Svaner de Hans Christian Andersen ", Féeries, $\mathrm{n}^{\circ}$ 9, "Le dialogue intertextuel des contes de Grimm», 20I2, p. $55-84$.

GIRARD René, Mensonge romantique et vérité romanesque [I977], Paris, Grasset, 200I.

Kelsen Hans, Théorie pure du droit, trad. H. Thévenaz, Neuchâtel, Éditions de la Baconnière, I988.

La Fontaine Jean de, Les Amours de Psyché et de Cupidon [I669], Fr. Charpentier (éd.), Paris, GF-Flammarion, 1990.

Pourrat Henri, Le Trésor des contes, Paris, Gallimard, I948, t. I. 
Sermain Jean-Paul, Le Conte de fées du classicisme aux Lumières, Paris, Desjonquères, 2005.

Solnon Jean-François, La Cour de France, Paris, Fayard, 1987.

Tatar Maria, The Hard Facts of the Grimms' Fairy Tales [1987], Princeton, Princeton University Press, 2003. 